Research Article

\title{
Preliminary Efficacy of Tocilizumab Treatment in the Patients with COVID-19
}

\author{
Yu Chen, Xijing Zhang* \\ Department of Critical Care Medicine, Xijing Hospital, Air Force Military Medical University, 15th Changle West Rd, Xian 710032, Shaanxi, China
}

\section{ARTICLE INFO}

\section{Article History}

Received 19 January 2021

Accepted 06 April 2021

\section{Keywords}

COVID-19

Tocilizumab

preliminary efficacy

safety

\begin{abstract}
Background: Interleukin-6 (IL-6) was considered to be with the severity and mortality in COVID-19 patients, which implies a potential therapeutic target for treatment. We aimed to evaluate the safety and initial efficacy of Tocilizumab treatment for COVID-19 patients.

Methods: In the retrospective study, 61 patients with COVID-19 with the mean age of 69 were enrolled from February 27 to March 14, 2020 in Wuhan Huoshenshan Hospital. Twenty-nine of them received one dose (400 mg) of add-on Tocilizumab treatment as the treated group and remaining 32 cases served as control group. The clinical manifestations and laboratory examinations were compared between the two groups.

Results: The average duration of symptoms appeal to hospital admission was 28.2 days. Compared with the cases in control group, the treated cases exhibited a significant increase of serum IL- 6 on the 7th day since Tocilizumab injection, however, there were no differences in whole blood white cell count, circulating lymphocyte count, serum C-reactive protein (CRP), and respiratory parameters or other clinical manifestations between the treated and control groups. There were no adverse events associated with Tocilizumab treatment in the treated COVID-19 patients.
\end{abstract}

Conclusion: In the patients with COVID-19, one dose of Tocilizumab treatment was safe but no clinical benefit was observed on the 7 th day in this study.

(C) 2021 First Affiliated Hospital of Zhengzhou University. Publishing services by Atlantis Press International B.V. This is an open access article distributed under the CC BY-NC 4.0 license (http://creativecommons.org/licenses/by-nc/4.0/).

\section{INTRODUCTION}

Global cases of confirmed COVID-19 have exceeded 88 million with $1,919,204$ deaths by 12 January 2021 [1]. The ongoing antiviral and immune modulation therapeutic regimens mainly include hydroxychloroquine, chloroquine, Favipiravir, Remdesivir, and convalescent plasma, mesenchymal stem cell therapy as well. However, most of them are lacky of evidence from randomized control clinical trials to recommend some specific and efficient therapeutic approaches. The pathogenesis of COVID-19 is not fully understood, the direct and indirect injuries induced by acute severe acute respiratory syndrome coronavirus 2 (SARS-CoV-2) infection influence the outcome of COVID-19. In particular, the indirect over-activated immune-mediated inflammatory injury was usually manifested by overactivation of peripheral T cells. Simultaneously, recent studies showed that interstitial mononuclear inflammatory infiltrates in lungs were both found in the patients [2]. Cytokine Storm Syndrome (CRS) often leads to the development of acute respiratory distress syndrome, or multiple organ dysfunction syndromes [3]. That clinical biomarkers such as interleukin-6 (IL-6), C-Reactive Protein (CRP), and ferritin, were also considered to be

"Corresponding author. Email: zhangxj918@163.com Peer review under responsibility of the First Affiliated Hospital of Zhengzhou University Data availability statement: The data that support the findings of this study are available from the corresponding author [XZ], upon reasonable request. with the severity and mortality in COVID-19 patients [4,5], which implies a potential therapeutic target for treatment of COVID-19. Tocilizumab, being a recombinant humanized monoclonal antibody directed against the Interleukin-6 Receptor (IL-6R), was previously issued to use in treatment of rheumatoid arthritis by American Food and Drug Administration (FDA) in 2010 [6]. In the epidemic of COVID-19, Tocilizumab was recommended to treat COVID-19 patients with increased IL-6 levels to block cytokine storm [7]. We hypothesize that the Tocilizumab as an additional immunotherapy may be associated with improved clinical outcome in the COVID-19 patients with elevated IL- 6 levels. Due to the difficulty of performing prospective randomized controlled studies in such epidemic condition, we reported the findings in this retrospective study to analyze the efficacy and safety of Tocilizumab in the patients with COVID-19.

\section{MATERIALS AND METHODS}

\subsection{Study Patients}

Sixty-one patients (40 males, 21 females) with COVID-19 were enrolled from February 27 to March 14, 2020 in Wuhan Huoshenshan Hospital, Hubei, China. Diagnostic criteria are based on the guideline of China's National Health Commission [7]. 
Clinical classification of moderate cases means the COVID-19 patients showing fever and respiratory symptoms with radiological findings of pneumonia [7]. Adult cases meeting any of the following criteria while patient was breathing ambient air at rest were diagnosed as severe type: (1) Dyspnea and respiratory frequency $\geq 30$ breaths/min; (2) Percutaneous oxygen saturation $\leq$ 93\%; (3) Arterial partial pressure of oxygen $\left(\mathrm{PaO}_{2}\right) /$ fraction of inspired oxygen $\left(\mathrm{FiO}_{2}\right) \leq 300 \mathrm{mmHg}$ [7]. Cases requiring mechanical ventilation, shock, with other organ failure that required ICU care were excluded [7]. This study was approved by the Ethics Committee of Wuhan Huoshenshan Hospital (Approval number: HSSLL033). Inclusion criteria included: (1) Diagnosed as COVID-19 (clinical classification of moderate or severe cases); (2) age > 18 years old; (3) IL-6 level $>7 \mathrm{pg} / \mathrm{mL}$. Exclusion criteria: (1) Pregnant or lactating women; (2) Alanine transaminase/aspartate transaminase $>$ five times upper limit of norm $(\mathrm{ULN})$, neutrophil $<0.5 \times 10^{9} / \mathrm{L}$, platelet $<50 \times 10^{9} / \mathrm{L}$; (3) Diagnosis of rheumatism immunity, malignant tumor, and other related diseases; (4) Long-term oral administration of antirejection drugs or immunomodulatory drugs; (5) Allergic reaction to Tocilizumab or any auxiliary materials; (6) Active hepatitis, tuberculosis; (7) Patients received organ transplantation; (8) Mental disorders. Serum IL-6 level were analyzed in 1086 patients in Wuhan Huoshenshan Hospital by March 13, the positive rate was $14.83 \%$ ( $>7 \mathrm{pg} / \mathrm{mL}$ ). Finally, 29 patients with COVID-19 treated with Tocilizumab in addition to conventional treatment. Thirty-two cases were enrolled in control group.

\subsection{Treatment Protocol}

In this retrospective study, all patients received a routine treatment protocol according to the guideline published by the China's National Health Commission [7], including antiviral drugs, symptom relievers, and oxygen therapy. Tocilizumab [Roche Pharma (Schweiz) Ltd., B2084B21] was solved in $100 \mathrm{~mL}$ normal saline. Only one dose of $400 \mathrm{mg}$ was given through an intravenous drip with infusion time more than $1 \mathrm{~h}$ [7]. The following outcomes were compared between groups: whole blood White Cell Count (WBC), lymphocyte count, level of IL-6 and CRP, peripheral oxygen saturations, body temperature, respiratory rate, respiratory support strategies, and length of hospital stay (Figure 1).

\subsection{Statistical Analysis}

All data, are expressed as mean $( \pm \mathrm{SD})$. Chi-square test or Fisher's exact test were used for categorical variables, Student's $t$-test or repeated-measures one-way analysis of variance with Tukey's post hoc test were used for continuous variables where appropriate.

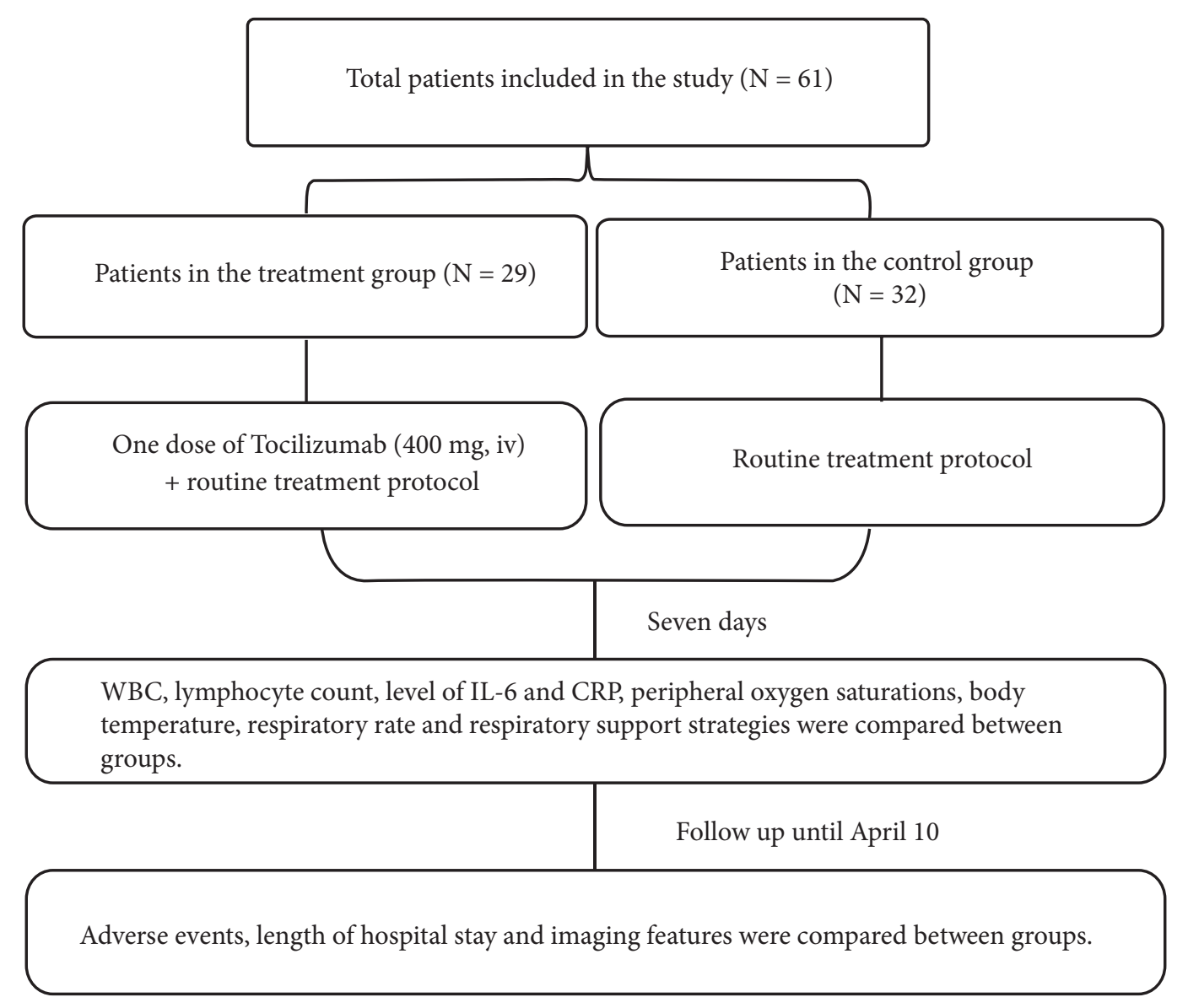

Figure 1 Flow chart. 
A two tailed $p$-value of $<0.05$ was considered significant. GraphPad Prism Version 8.4.0 (GraphPad Software Inc., San Diego, CA, USA) was used for all analyses.

\section{RESULTS}

\subsection{Demographic Characteristics}

The 61 patients had a mean $( \pm \mathrm{SD})$ age of $69( \pm 13.1)$ years, ranged from 19 to 93 years and there were $40(65.6 \%)$ men. Mean duration $( \pm \mathrm{SD})$ of symptoms appeal to hospital admission was $28.2( \pm 13.3)$ days $(29.4 \pm 13.7$ days in the control group, $27.0 \pm 13.0$ days in the treated group). Fifty-one patients (83.6\%) with the course of the disease over 2 weeks. Thirty-four patients (55.7\%) met moderate type criteria, and $27(44.3 \%)$ met severe type criteria according to the "New Coronavirus Pneumonia Diagnosis and Treatment Plan (Seven Edition)" [7]. Thirty-six patients (59.0\%) had at least a chronic co-existing illness. Twenty-nine patients (47.5\%) received one dose of Tocilizumab as treatment group and the others were assigned to the control group. After admission, all patients needed an oxygen therapy, including nasal cannula in 35 patients (57.4\%), mask oxygen in 15 patients $(24.6 \%)$, high-flow nasal oxygen therapy in 11 patients $(18.0 \%)$, and no patient received noninvasive ventilation or invasive ventilation. The baseline data of enrolled patients were shown in Table 1. A significant difference was not found between the cases of two groups.

Table 1 Demographic characteristics of enrolled patients

\begin{tabular}{|c|c|c|c|}
\hline Characteristics & $\begin{array}{c}\text { Treatment } \\
\text { group }(N=29)\end{array}$ & $\begin{array}{l}\text { Control group } \\
\qquad(N=32)\end{array}$ & $p$-value \\
\hline Age (mean $\pm S D$, years) & $68.5 \pm 11.3$ & $69.4 \pm 15.8$ & 0.8 \\
\hline Male/Female & $22 / 7$ & $18 / 14$ & 0.11 \\
\hline Existing illness & 17 & 19 & $>0.99$ \\
\hline $\mathrm{AD}$ & 0 & 1 & - \\
\hline Anemia & 1 & 1 & - \\
\hline $\mathrm{CHD}$ & 9 & 7 & - \\
\hline CKD & 1 & 1 & - \\
\hline CLD & 1 & 1 & - \\
\hline COPD & 0 & 4 & - \\
\hline Diabetes & 8 & 10 & - \\
\hline Hypertension & 11 & 8 & - \\
\hline State of illness & - & - & 0.31 \\
\hline Mild or moderate & 14 & 20 & - \\
\hline Severe & 15 & 12 & - \\
\hline Temperature $\left(\right.$ mean $\left.\pm \mathrm{SD},{ }^{\circ} \mathrm{C}\right)$ & $36.7 \pm 0.4$ & $36.5 \pm 0.3$ & 0.06 \\
\hline $\mathrm{RR}($ mean $\pm \mathrm{SD}, \mathrm{bpm})$ & $20.1 \pm 2.2$ & $20.0 \pm 1.4$ & 0.83 \\
\hline $\mathrm{SpO}_{2}($ mean $\pm \mathrm{SD}, \%)$ & $96.1 \pm 2.6$ & $96.3 \pm 2.6$ & 0.79 \\
\hline Oxygen therapy & - & - & 0.98 \\
\hline Nasal cannula (<3 L/min) & 17 & 18 & - \\
\hline Mask oxygen & 7 & 8 & - \\
\hline High-flow oxygen & 5 & 6 & - \\
\hline Noninvasive ventilation & 0 & 0 & - \\
\hline Invasive ventilation & 0 & 0 & - \\
\hline $\begin{array}{l}\text { Duration of symptoms to } \\
\text { admission (mean } \pm \text { SD, days) }\end{array}$ & $27.0 \pm 13.0$ & $29.4 \pm 14.0$ & 0.48 \\
\hline $\begin{array}{l}\text { Hospitalization } \\
\qquad(\text { mean } \pm \mathrm{SD} \text {, days })\end{array}$ & $6.3 \pm 7.0$ & $3.5 \pm 6.2$ & 0.1 \\
\hline
\end{tabular}

$\mathrm{AD}$, Alzheimer disease; $\mathrm{CHD}$, coronary heart disease; $\mathrm{CKD}$, chronic kidney disease; CLD, chronic liver disease; COPD, chronic obstructive pulmonary disease.

\subsection{Adverse Events}

Adverse drug reaction such as decreased blood pressure, nausea, dizziness and rash of Tocilizumab were observed. No adverse drug reaction related to Tocilizumab was reported. All patients survived in this study. There was no difference in the days of hospitalization between groups (Table 2). Patients given Tocilizumab had higher blood IL-6 levels at 7th day (Figure 2). Between Tocilizumab treated patients with serum IL-6 levels above and below $100 \mathrm{pg} / \mathrm{mL}$ at 7 th day, the length of hospital stays showed no differences $(26 \pm 13.2$ vs. $26 \pm 10.7, p>0.05$, shown as mean \pm SD days). Until April 15, 2020, 68 patients were discharged from hospital, one Tocilizumab treated patient was transferred to another specialized hospital for further treatment. His hospital stay was record as 33 days in Wuhan Huoshenshan Hospital.

\subsection{No Difference of Clinical Presentations between Treated and Control Groups}

On the median day 7 , the changes of clinical manifestations and laboratory examinations between the two groups were compared. All patients return to normal temperature $\left(<37.3^{\circ} \mathrm{C}\right.$, axillary temperature), mean Respiratory Rate (RR) and oxyhemoglobin saturation by pulse oximetry $\left(\mathrm{SpO}_{2}\right)$ were also in a normal range. Respiration support was de-escalated, including nasal cannula in 53 patients (86.9\%), mask oxygen in two patients $(3.3 \%)$, high-flow nasal oxygen therapy in six patients $(9.8 \%)$. There was no difference of body temperature, $\mathrm{RR}, \mathrm{SpO}_{2}$, or the way of oxygen therapy between the two groups (Table 2).

Interleukin-6 expression levels were analyzed in all patients on day 1 defined as the first day of detectable increasement of IL-6 with the mean $\pm \mathrm{SD}$ of $32.6 \pm 43.9 \mathrm{pg} / \mathrm{mL}$ in control group and $29.4 \pm 46.1 \mathrm{pg} / \mathrm{mL}$ in the treatment group (Figure 2A). After 7 days, mean IL-6 expression levels were significantly upregulated in the treatment group as compared with control group $(p<0.01$, Figure 2A). White blood cell count showed a decrease in both groups as compared with the level on day $1(p<0.05)$. But there was no difference between the two groups at the same time point (Figure $2 \mathrm{~B}$ ). Compared with the level on day 1, CRP levels on day 7 were decreased in both groups. No difference was found between the two groups at the same time point (Figure 2C). There was no difference in lymphocyte count between groups at any time (Figure 2D).

Table 2 Comparison of parameters between treated and control group at the 7 th day of Tocilizumab treatment

\begin{tabular}{lccc}
\hline Characteristics & $\begin{array}{c}\text { Treatment } \\
\text { group }(\boldsymbol{N}=\mathbf{2 9})\end{array}$ & $\begin{array}{c}\text { Control group } \\
(\boldsymbol{N}=\mathbf{3 2})\end{array}$ & $\boldsymbol{p}$-value \\
\hline Temperature $\left(\right.$ Mean $\left.\pm \mathrm{SD},{ }^{\circ} \mathrm{C}\right)$ & $36.5 \pm 0.2$ & $36.5 \pm 0.2$ & 0.53 \\
$\mathrm{RR}($ Mean $\pm \mathrm{SD}, \mathrm{bpm})$ & $19.0 \pm 1.6$ & $19.2 \pm 1.4$ & 0.63 \\
$\mathrm{SpO}_{2}$ (Mean $\left.\pm \mathrm{SD}, \%\right)$ & $97.8 \pm 1.3$ & $97.9 \pm 0.9$ & 0.78 \\
Oxygen therapy & - & - & 0.99 \\
$\quad$ Nasal cannula $(<3 \mathrm{~L} / \mathrm{min})$ & 25 & 28 & - \\
$\quad$ Mask oxygen & 1 & 1 & - \\
$\quad$ High-flow oxygen & 3 & 3 & - \\
Length of hospital stay & $22.1 \pm 9.5$ & $26 \pm 11.3$ & 0.15 \\
$\quad($ Mean $\pm \mathrm{SD}$, days) & & & - \\
Death & 0 & 0 & - \\
\hline
\end{tabular}



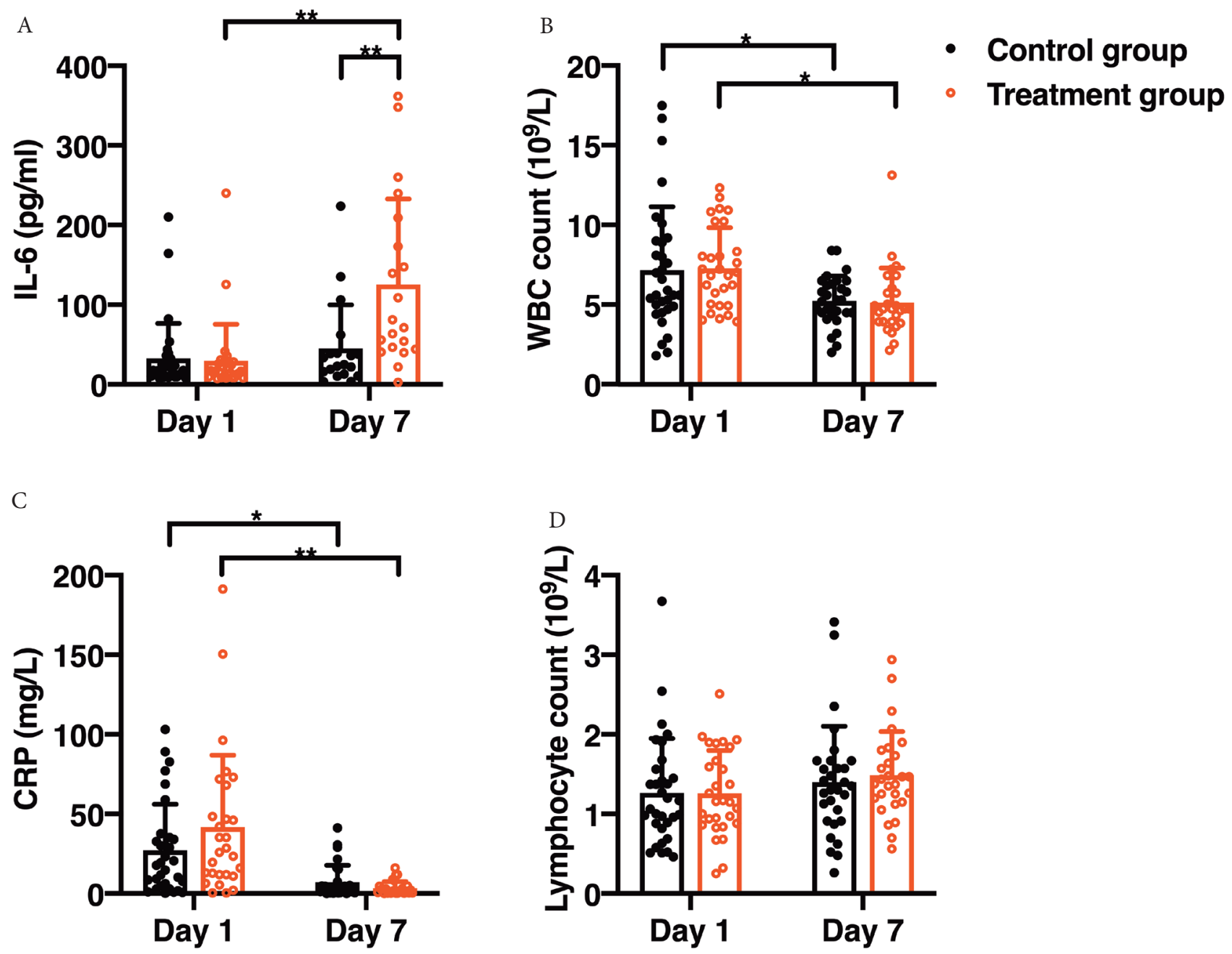

Figure 2 The values of IL-6, CRP, WBC and lymphocyte count between groups. Day 1 , the time of first detectable IL- 6 elevation. ${ }^{*} p<0.05,{ }^{* *} p<0.01$. (A) After 7 days, mean \pm SD IL-6 expression levels were upregulated in the treatment group as compared with control group (125.5 \pm 107.3 vs. $45.1 \pm$ $54.9 \mathrm{pg} / \mathrm{mL})$. (B) White blood cell count showed a decrease in both groups as compared with the level on day $1\left(5.2 \pm 1.5 \mathrm{vs} .7 .2 \pm 3.9 \times 10^{9} / \mathrm{L}\right.$ in control group ( $5.1 \pm 2.1$ vs. $7.3 \pm 2.5 \times 10^{9} / \mathrm{L}$ in treatment group). There was no difference between the two groups at the same time point. (C) Compared with the level on day 1, CRP levels on day 7 were decreased in both groups. ( $7.0 \pm 10.7 \mathrm{vs.} 27.3 \pm 28.8 \mathrm{mg} / \mathrm{L}$ in control group. $3.4 \pm 4.3 \mathrm{vs.} 41.8 \pm 45.1 \mathrm{mg} / \mathrm{L}$ in treatment group) and no difference was found between the two groups at the same time point. (D) There was no difference in lymphocyte count between groups at any time.

\subsection{Imaging Features were Similar in the Two Groups}

Varying degrees of consolidations or mixed ground-glass opacities were presented by chest computerized tomography (CT, uCT760, United Imaging Co., Beijing, China) in most patients (Figure 3). Differences of percentage of infection were calculated by a modified $3 \mathrm{D}$ convolutional neural network-based method, the values $<1.0 \%$ were not included for the observational error. Imaging features were followed up until April 10. Seventeen patients in the control group and 18 patients in the Tocilizumab treatment group were performed at least two CT scans for comparison. The mean time interval between inspections was 17.7 days (17.5 \pm 8.8 days in the control group, $17.9 \pm 11.3$ days in the Tocilizumab treatment group, shown as mean \pm SD). Different levels of lesions absorption were reported in 11 patients of Tocilizumab treatment group (61.1\%) and 10 patients in the control group (58.8\%). There were no differences between groups at any time (Figure 4).

\section{DISCUSSION}

Inflammatory cytokines are elevated COVID-19 patients, and related to the severity and prognosis of the disease [8,9]. Histological examination also shown mononuclear inflammatory lymphocytes were infiltrated in lungs [8], suggesting an inflammatory factor or a CRS have occurred. Severe or life-threatening CRS is a medical emergency requiring treatments includes fluids resuscitation and corticosteroids in some patients, which may cause further deterioration of lung function and aggravation of infection [10]. Several cytokines including IL- 6 are participated in the pathological process of CRS, and immunosuppression using Tocilizumab, an anti-IL-6R antibody, can reverse the syndrome $[11,12]$. Previous study indicated that an elevated level of IL- 6 was associated with a high case fatality of COVID-19 patients [13]. And circulating IL-6 levels were closely linked to the severity of COVID-19 infection in a recent meta-analysis [5]. Therefore, in theory, interfering of IL-6R with Tocilizumab might be a potentially therapeutic for COVID-19. 
A 75-year-old male patient in the control group.

\section{An 81-year-old male patient in the treatment group.}
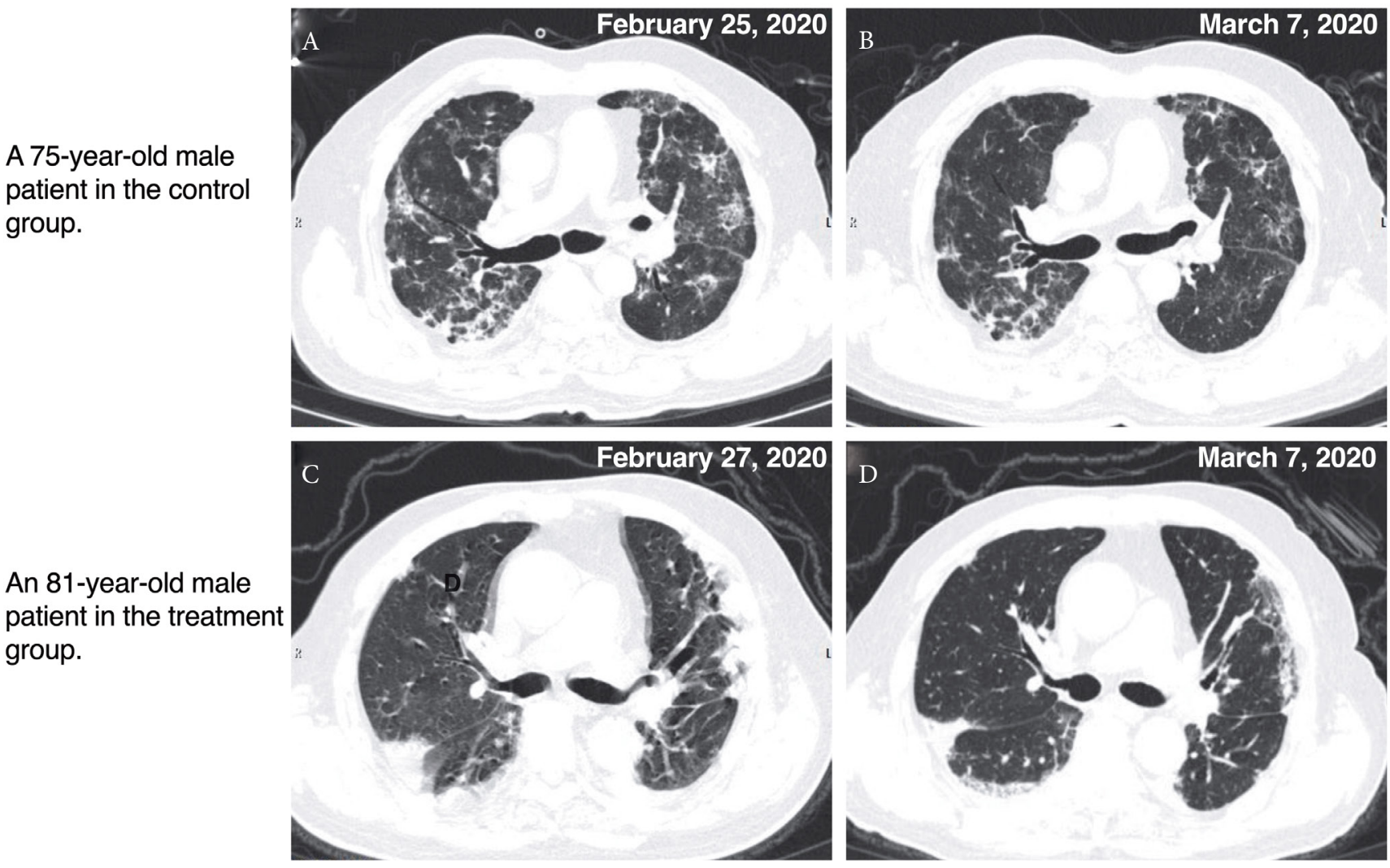

Figure 3 Chest CT imaging images. The CT image (A) of a 75-year-old male patient in the control group was performed at February 25, patchy and punctate ground-glass opacities and consolidations could be observed. CT scan was performed again at March 7, the density of patchy and punctate consolidation decreased (B). The CT image of an 81-year-old male patient in the treatment group (C) was performed at February 27, consolidation in the subpleural area could be found. CT scan was performed again at March 7, the density and range of consolidation decreased (D).

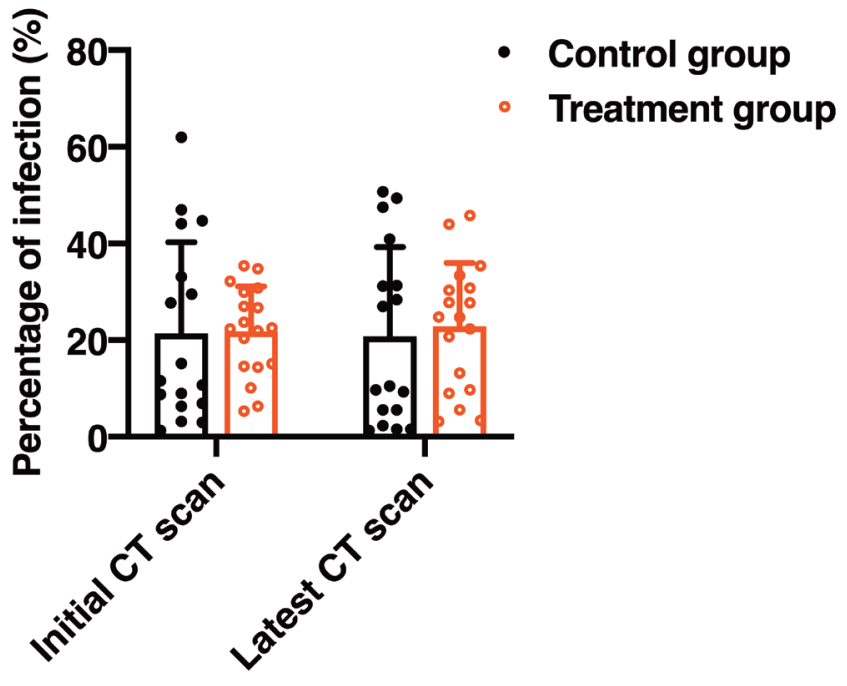

Figure 4 The values of percentage of infection calculated by an artificial intelligence (AI) method from CT reports between groups. Mean time interval between inspections (initial and latest CT scan) was 17.7 days $(17.5 \pm 8.8$ days in the control group, and $17.9 \pm 11.3$ days in the Tocilizumab treatment group, shown as mean \pm SD). The mean \pm SD infection percentage of initial CT scan and latest CT scan was $21.3 \pm$ $18.8 \%$ and $20.7 \pm 18.4 \%$ respectively in the control group, and $21.8 \%$ $\pm 9.3 \%$ and $22.8 \% \pm 13.2 \%$ in the treatment group. There were no differences between groups at any time.
Tocilizumab was used to treat rheumatoid arthritis [6]. The latest guideline issued by Chinese National Health Commission recommended Tocilizumab for treatment of COVID-19 patients with increased IL-6 levels [7]. Serum IL-6 level were analyzed in 1,086 patients in Wuhan Huoshenshan Hospital by March 13, the positive rate was $14.83 \%(>7 \mathrm{pg} / \mathrm{mL})$, and 29 patients treated with Tocilizumab were enrolled in this study.

Tocilizumab binds both soluble and membrane-bound IL-6R and inhibits IL-6 mediated signaling through these receptors without increasing half-life of IL-6 [10]. Clearance of single-dose Tocilizumab was slow, with a half-life more than 6 days in both rats and monkeys [14]. In human, after the third dose of $8 \mathrm{mg} / \mathrm{kg}$, its maximum halflife nearly $240 \mathrm{~h}$. So, it can be administered biweekly or monthly [15]. In our study, one dose $400 \mathrm{mg}$ Tocilizumab was associated with a rebound IL-6 upregulation on the median day 7. Which is reasonable as that Tocilizumab has a long elimination half-life time, the block of IL-6R would stimulate more IL-6 expression [16].

Unfortunately, no clinical benefit of Tocilizumab in treatment of 29 patients with moderate or severe COVID-19 in this retrospective study. One explanation is that the illness of patients included in this study is relatively mild. By 18 January 2021, there were 88,454 confirmed cases and 4635 deaths have been reported in China, the mortality was nearly 5.2\% [17], and according to a new report, the mortality of critical cases reached $60.5 \%$ [18]. While all the patients survive during the entire observation period in our study. The recommendation of Tocilizumab was mentioned in the latest guideline 
published in March 4 [7]. Before that, IL-6 was not routine tested and the clinicians were more willing to try additional Tocilizumab treatment in relatively mild patients to avoid any uncertain factors in critical cases.

In the pathogenesis of SARS-CoV and middle east respiratory syndrome coronavirus (MERS-CoV), cytokine genes of IL-6 can be markedly high $[19,20]$. One research also found elevated plasma levels of cytokines including IL-6 in patients with COVID-19 [21]. In our study, the baseline CRP levels were $<100 \mathrm{mg} / \mathrm{L}$ in both groups, indicting the inflammatory reaction may not be serious in these patients. Which could minimize the clinical benefits of Tocilizumab treatment. However, the elevation of IL-6 was not observed in a recent study [9]. Indicating a second and probably a more important possibility, that IL-6 may not play a key role in the pathogenesis of COVID-19, even in the cases with obvious inflammatory response.

Many reasons could explain our frustrating results, including small sample size, differences in baseline levels, lack of IL-6 data, and failure to include critically ill patients. We acknowledge these shortcomings and expect further well-designed RCT trails looking at timing of treatment intervention and type of aged patients to draw a more rigorous conclusion. Meanwhile, COVID-19 patients may experience life-threatening CRS which may cause severe damage to various systems. It is wise to provide effective organ support and carefully prevent complications, instead of expecting unproved miracles.

\section{CONCLUSION}

In summary, in the COVID-19 patients, one dose Tocilizumab treatment was safe but no clinical benefit was demonstrated on the 7 th day in this study.

\section{CONFLICTS OF INTEREST}

The authors declare they have no conflicts of interest.

\section{AUTHORS' CONTRIBUTION}

$\mathrm{Yu}$ Chen gathered the data, conducted the statistical analyses and drafted the manuscript and figures. Xijing Zhang and Yu Chen conceived the concept of the study and supervised data collection. The authors critically reviewed and validated the final version of the manuscript.

\section{FUNDING}

This work was supported by the Natural Science Foundation of Shaanxi Province of China (2019JM-465) and Subject Boosting Program of Air Force Military Medical University (XJZT18ML63).

\section{ETHICAL APPROVAL AND CONSENT TO PARTICIPATE}

This study was approved by the Ethics Committee of Wuhan Huoshenshan Hospital (Approval number: HSSLL033). All written consents from patients were waived due to the retrospective nature of the current study.

\section{TRIAL REGISTRATION}

ChiCTR2000033705, June 10, 2020, retrospectively registered.

\section{REFERENCES}

[1] World Health Organization. Coronavirus disease (COVID- 19) situation reports. [Internet]. 2020. Available from: https:// www.who.int/emergencies/diseases/novel-coronavirus-2019/ situation-reports.

[2] Xu Z, Shi L, Wang Y, Zhang J, Huang L, Zhang C, et al. Pathological findings of COVID-19 associated with acute respiratory distress syndrome. Lancet Respir Med 2020;8;420-2.

[3] Tisoncik JR, Korth MJ, Simmons CP, Farrar J, Martin TR, Katze MG. Into the eye of the cytokine storm. Microbiol Mol Biol Rev 2012;76;16-32.

[4] Ruan Q, Yang K, Wang W, Jiang L, Song J. Clinical predictors of mortality due to COVID-19 based on an analysis of data of 150 patients from Wuhan, China. Intensive Care Med 2020;46;846-8.

[5] Ulhaq ZS, Soraya GV. Interleukin-6 as a potential biomarker of COVID-19 progression. Med Mal Infect 2020;50;382-3.

[6] June RR, Olsen NJ. Room for more IL-6 blockade? Sarilumab for the treatment of rheumatoid arthritis. Expert Opin Biol Ther 2016;16;1303-9.

[7] China's National Health Commission. Diagnosis and treatment protocol for novel coronavirus pneumonia. [Internet] 2020; Trial Version 7. Available from: http://www.nhc.gov.cn/yzygj/ s7653p/202003/46c9294a7dfe4cef80dc7f5912eb1989.shtml.

[8] Chen N, Zhou M, Dong X, Qu J, Gong F, Han Y, et al. Epidemiological and clinical characteristics of 99 cases of 2019 novel coronavirus pneumonia in Wuhan, China: a descriptive study. Lancet 2020;395;507-13.

[9] Huang C, Wang Y, Li X, Ren L, Zhao J, Hu Y, et al. Clinical features of patients infected with 2019 novel coronavirus in Wuhan, China. Lancet 2020;395;497-506.

[10] Le RQ, Li L, Yuan W, Shord SS, Nie L, Habtemariam BA, et al. FDA approval summary: tocilizumab for treatment of chimeric antigen receptor $\mathrm{T}$ cell-induced severe or life-threatening cytokine release syndrome. Oncologist 2018;23;943-7.

[11] Teachey DT, Lacey SF, Shaw PA, Melenhorst JJ, Maude SL, Frey N, et al. Identification of predictive biomarkers for cytokine release syndrome after chimeric antigen receptor T-cell therapy for acute lymphoblastic leukemia. Cancer Discov 2016;6;664-79.

[12] Lee DW, Gardner R, Porter DL, Louis CU, Ahmed N, Jensen M, et al. Current concepts in the diagnosis and management of cytokine release syndrome. Blood 2014;124;188-95.

[13] Chen X, Zhao B, Qu Y, Chen Y, Xiong J, Feng Y, et al. Detectable serum severe acute respiratory syndrome coronavirus 2 viral load (RNAemia) is closely correlated with drastically elevated interleukin 6 level in critically ill patients with coronavirus disease 2019. Clin Infect Dis 2020;71;1937-42.

[14] Jones G, Ding C. Tocilizumab: a review of its safety and efficacy in rheumatoid arthritis. Clin Med Insights Arthritis Musculoskelet Disord 2010;3;81-9.

[15] Nishimoto N, Yoshizaki K, Maeda K, Kuritani T, Deguchi H, Sato $\mathrm{B}$, et al. Toxicity, pharmacokinetics, and dose-finding study of 
repetitive treatment with the humanized anti-interleukin 6 receptor antibody MRA in rheumatoid arthritis. Phase I/II clinical study. J Rheumatol 2003;30;1426-35.

[16] Frey N, Grange S, Woodworth T. Population pharmacokinetic analysis of tocilizumab in patients with rheumatoid arthritis. J Clin Pharmacol 2010;50;754-66.

[17] National Health Commission of the People's Republic of China. Epidemic notification for COVID-19. [Internet]. 2020. Available from: http://www.nhc.gov.cn/xcs/yqtb/list_gzbd.shtml.

[18] Yang X, Yu Y, Xu J, Shu H, Xia J, Liu H, et al. Clinical course and outcomes of critically ill patients with SARS-CoV-2 pneumonia in Wuhan, China: a single-centered, retrospective, observational study. Lancet Respir Med 2020;8;475-81.
[19] Li Y, Chen M, Cao H, Zhu Y, Zheng J, Zhou H. Extraordinary GU-rich single-strand RNA identified from SARS coronavirus contributes an excessive innate immune response. Microbes Infect 2013;15;88-95.

[20] Lau SKP, Lau CCY, Chan KH, Li CPY, Chen H, Jin DY, et al. Delayed induction of proinflammatory cytokines and suppression of innate antiviral response by the novel Middle East respiratory syndrome coronavirus: implications for pathogenesis and treatment. J Gen Virol 2013;94;2679-90.

[21] Zhou Y, Fu B, Zheng X, Wang D, Zhao C, Qi Y, et al. Pathogenic T-cells and inflammatory monocytes incite inflammatory storms in severe COVID-19 patients. Natl Sci Rev 2020;7; 998-1002. 